\title{
Relativistic description of electron scattering on the deuteron
}

\author{
E. Hummel* and J. A. Tjon \\ Institute for Theoretical Physics, University of Utrecht, \\ Princetonplein 5, 3508 TA Utrecht, The Netherlands
}

\begin{abstract}
Within a quasipotential framework a relativistic analysis is presented of the deuteron current. Assuming that the singularities from the nucleon propagators are important, a so-called equal time approximation of the current is constructed. This is applied to both elastic and inelastic electron scattering. As dynamical model the relativistic one boson exchange model is used. Reasonable agreement is found with a previous relativistic calculation of the elastic electromagnetic form factors of the deuteron. For the unpolarized inelastic electron scattering effects of final state interactions and relativistic corrections to the structure functions are considered in the impulse approximation. Two specific kinematic situations are studied as examples.
\end{abstract}

PACS numbers: 11.10.Qr, 25.30-c, 25.30.Bf

\section{INTRODUCTION}

Electron scattering processes have been crucial in extracting detailed information about the nuclear interaction. Various exclusive [1,2, [3] and inclusive [4,5] breakup reactions for the few nucleon systems have recently for example been performed at moderate momentum transfer, where separations of the longitudinal and transverse response functions have successfully been achieved. The deuteron, as the most simple nuclear system, is of special interest because exact calculations are in principle feasible for this case. Such a system may serve as testing ground for theoretical models describing the nuclear dynamics and the e.m. operators used to study these reaction processes. In most of these studies it is implicitly assumed that the constituents of the system behave nonrelativistically and that the e.m. interaction can be treated in an independent way. With increasing momentum and energy transfer effects of relativity are expected to play an important role and a relativistic description should be essential in these kinematic regions. Moreover, in view of gauge invariance a consistent treatment of the e.m. interaction is called for.

In this paper we discuss a framework which is well suited for a relativistic analysis of both the elastic and inelastic electron scattering off the deuteron. It is based on the relativistically covariant field theoretical Bethe-Salpeter equation approach. Gauge invariance is satisfied through the Ward-Takahashi identity. Assuming a theoretical description in terms of nucleonic and mesonic degrees of freedom the elastic e.m. properties have already been studied. Within an one boson exchange (OBE) model [6] a consistent relativistic treatment of both the e.m. current and the nucleonnucleon interaction can be realized [7], including also meson exchange current( MEC) contributions from the $\rho \pi \gamma$ and $\omega \varepsilon \gamma$ currents [8]. In the actual calculations a relativistic quasipotential approximation of the Blankenbecler-SugarLogunov-Tavkhelidze (BSLT) has been used.

The disintegration experiments of the deuteron yields additional information as compared to the elastic e.m. scattering process. A larger kinematic region can be tested and the theoretical models are more complicated due to final state interaction (FSI) and other MEC contributions as is the case in elastic scattering. Another point of interest is that, depending on the type of experiment, the cross section can be separated in more than 2 structure functions. The inclusive experiment is described by the longitudinal $R_{L}$ and transverse $R_{T}$ structure functions. For the exclusive experiment studied here an additional structure function $R_{T L}$ is measured, which is an interference between the longitudinal and transverse components in the current operator. Because this structure function depends on other reaction processes as $R_{L}$ and $R_{T}$, new information can be obtained.

In our calculations complete knowledge of the FSI and the deuteron wave function is required. As mentioned earlier we use the OBE model of Ref. [6]. Although the complete model can be studied we use here a quasipotential approximation to it, where both particles are treated in a symmetrical way. This BSLT approximation [9,10] was also used in the model of the elastic form factors. Here we'll use the same approximation for the initial and final state, but use a different approximation for the nucleon propagators occurring in the e.m. vertex. We assume that only a relative energy dependence is in these propagators and not in the two-nucleon vertex functions. As a result the relative energy dependence can explicitly be integrated out. Because the BSLT choice is used for the nucleon states 
this is an equal time (ET) approximation to only the current operator. To examine the sensitivity of the predictions on such a ET prescription for the e.m. current matrix elements we calculate the e.m. form factors in the elastic case. Comparing to earlier calculations [8] we find only small differences. The ET choice has the advantage that it can also be extended in a systematic way to describe the case of inelastic electron scattering. In the full Bethe-Salpeter theory a conserved deuteron current can be constructed (see Refs. [7.11]), at least for the case of strong form factors at the meson-nucleon vertices, which depend only on the momentum of the meson. The same can be achieved in a systematic way in the ET approximation, i.e. MEC contributions can be constructed for the breakup reaction to ensure current conservation.

The paper is organized as follows. In the next two sections we summarize the relevant expressions, characterizing the inelastic electron scattering processes and describe the dynamical model used in this work. In particular, we present a new fit in this model to the experimental phase shifts, to discuss the effect of including the negative energy states. In sections IV and V the relativistic impulse approximation and the elastic e.m. form factor calculations are described in the ET approximation. Section VI deals with the formalism of the e.m. breakup of the deuteron in this approximation. In all these calculations a fully relativistic e.m. current operator with on shell form factors is used. In section VII two kinematic situations are studied using this relativistic formalism. For the conventional nonrelativistic (NR) models different forms of e.m. operators have been constructed in the literature. These models differ predominantly in the use of $F_{1}$ or $G_{E}$ for the e.m. nucleon form factors and the differences are obviously of relativistic order. We compare these predictions with our fully relativistic analysis and show that in the kinematics studied the predictions can be very different. Most of the differences can be traced back to the choice of the e.m. operator. Although the experiments are at moderate momentum transfer, large relativistic effects are in particular found in the interference structure function $R_{T L}$, showing the failure of the NR models discussed here. The next leading order correction to these operators is given and shown to represent reasonably well the relativistic predictions, at least in the kinematics considered. In the kinematic situations discussed in this section the MEC contributions which are needed to ensure gauge invariance are of minor importance. In a next paper we will discuss in detail our MEC calculations in this relativistic model.

\section{DEUTERON STRUCTURE FUNCTIONS}

In this section we summarize some useful formulae describing the electrodisintegration process of the deuteron. The conventions of Björken and Drell [12 are used. We confine ourselves in this paper to the description of unpolarized electron scattering on an unpolarized target and follow closely the work of Donnelly and Raskin [13]. The analysis can readily be extended to the case of polarized scattering.

For definiteness, let us consider the breakup of a deuteron with total momentum $P$ by an electron into a free neutron-proton (np) pair, characterized by the four momenta $p_{n}=\left(\mathbf{p}_{n}, E_{n}\right)$ and $p_{p}=\left(\mathbf{p}_{p}, E_{p}\right)$ respectively. In the one-photon exchange approximation, the differential cross section in the lab system, being the rest frame of the deuteron i.e. $P=\left(\mathbf{0}, M_{D}\right)$, can be written as [12]

$$
d \sigma=C_{n p} \frac{\varepsilon}{|\mathbf{k}|} \sum_{i f}^{-} \frac{m_{e}^{2}}{\varepsilon^{\prime} \varepsilon} \frac{d^{3} k^{\prime}}{(2 \pi)^{5}} d^{3} p_{p} d^{3} p_{n} \delta^{4}\left(k+P-k^{\prime}-P^{\prime}\right) \frac{e^{4}}{q_{\mu}^{4}}\left|j_{\mu}^{e} J^{\mu}\right|^{2},
$$

with $C_{n p}=M_{N}^{2} /\left(E_{n} E_{p}\right)$ and where $j_{\mu}^{e}$ is the electron and $J^{\mu} \equiv<f\left|J_{d}^{\mu}\right| i>$ the deuteron electromagnetic current matrix element between the initial and final state of the nuclear system. The total four momentum of the np pair is assumed to be given by $P^{\prime}$, while $q$ is the four momentum of the virtual photon satisfying $q_{\mu}=k_{\mu}-k_{\mu}^{\prime} \equiv(\mathbf{q}, \omega)$, $k_{\mu}=(\mathbf{k}, \varepsilon)$ and $k_{\mu}^{\prime}=\left(\mathbf{k}^{\prime}, \varepsilon^{\prime}\right)$ being the four momenta of the incoming and scattered electron. Momentum conservation at the photon-deuteron vertex gives $P+q=P^{\prime}$. The normalization of the outgoing np pair state is such that when the final state interaction is neglected it is given by $\left\langle p_{p}, p_{n}\right| f>\approx u\left(\mathbf{p}_{p}\right) u\left(\mathbf{p}_{n}\right), u$ being Björken-Drell spinors. In

Eq. (2.1) $\sum_{i f}^{-}$indicates averaging and summing over both the electron and nuclear polarizations in the initial and final state respectively. An electron and hadronic current tensor

$$
\eta_{\mu \nu}=\sum_{i f}^{-} j_{\mu}^{e^{*}} j_{\nu}^{e}, W_{\mu \nu}=C_{n p} \sum_{i f}^{-} J_{\mu}^{*} J_{\nu},
$$

can now be introduced such that $\sum_{i f}^{-}\left|j_{\mu}^{e} J^{\mu}\right|^{2}=\eta_{\mu \nu} W^{\mu \nu}$. The expression for $\eta_{\mu \nu}$ is well known [12] and is given in the 
ultra relativistic limit $\left(m_{e} \approx 0\right)$ by

$$
\eta_{\mu \nu}=\frac{1}{2 m_{e}^{2}}\left(k_{\mu} k_{\nu}^{\prime}+k_{\nu} k_{\mu}^{\prime}-g_{\mu \nu} k \cdot k^{\prime}\right) .
$$

Introducing the relative momentum $\mathbf{p}^{\prime}=\left(\mathbf{p}_{p}-\mathbf{p}_{n}\right) / 2$ of the np pair and integrating over $\mathbf{P}^{\prime}$ yields for the cross section

$$
d \sigma=\frac{m_{e}^{2}}{|\mathbf{k}| \varepsilon^{\prime}} \frac{e^{4}}{q_{\mu}^{4}} \frac{d^{3} k^{\prime} d^{3} p^{\prime}}{(2 \pi)^{5}} \delta\left(M_{D}+\omega-E_{p}-E_{n}\right) \eta_{\mu \nu} W^{\mu \nu} .
$$

At this point the concept of the structure functions can be introduced. We may choose a coordinate system, where $\mathbf{q}$ is along the $z$-axis, while the $y$-axis is defined along $\mathbf{k} \times \mathbf{k}^{\prime}$. Let us now define in this lab frame $J_{ \pm}=\mp \frac{1}{\sqrt{2}}\left(J_{x} \pm i J_{y}\right)$, and use current conservation $q \cdot J=0$ to eliminate the longitudinal third component $J_{3}$ of the current in favor of the charge $J_{3}=\omega / q J_{0}$. With the help of Eq. (2.3) the contraction can be written in the form

$$
\eta_{\mu \nu} W^{\mu \nu}=v_{0}\left(v_{L} R_{L}+v_{T} R_{T}+v_{T T} R_{T T}+v_{T L} R_{T L}\right)
$$

with $v_{0}=4 \varepsilon \varepsilon^{\prime} \cos ^{2} \frac{1}{2} \theta_{e}, \theta_{e}$ being the scattered electron angle. Moreover, the electron-kinematic factors are given by $v_{L}=\left(\frac{q_{\mu}^{2}}{\mathbf{q}^{2}}\right)^{2}, v_{T}=-\frac{1}{2} \frac{q_{\mu}^{2}}{\mathbf{q}^{2}}+\tan ^{2} \frac{1}{2} \theta_{e}, v_{T T}=\frac{1}{2} \frac{q_{\mu}^{2}}{\mathbf{q}^{2}}$ and $v_{T L}=\frac{1}{\sqrt{2}} \frac{q_{\mu}^{2}}{\mathbf{q}^{2}} \sqrt{-\frac{q_{\mu}^{2}}{\mathbf{q}^{2}}+\tan ^{2} \frac{1}{2} \theta_{e}}$. The nuclear structure functions $R_{\alpha}$ can be expressed in the matrix elements of the various current components as [13]

$$
\begin{aligned}
R_{L} & =C_{n p}\left|J_{0}\right|^{2}, \\
R_{T} & =C_{n p}\left(\left|J_{+}\right|^{2}+\left|J_{-}\right|^{2}\right), \\
R_{T T} & =2 C_{n p} \operatorname{Re}\left(J_{+}^{*} J_{-}\right), \\
R_{T L} & =-2 C_{n p} \operatorname{Re}\left[J_{0}^{*}\left(J_{+}-J_{-}\right)\right] .
\end{aligned}
$$

It should be noted that $C_{n p}$ can be absorbed in the Björken-Drell spinors occurring in the e.m. current matrix elements. The resulting spinors become essentially those defined by Kubis [14], described in Appendix A. In the remainder of the paper these spinors will be used. Introducing the Mott cross section $\sigma_{M o t t}=\frac{\alpha^{2}}{q_{\mu}^{4}} \frac{\varepsilon^{\prime}}{\varepsilon} v_{0}$, with the fine structure constant given by $\alpha=e^{2} /(4 \pi)$, the cross section can be written as

$$
d \sigma=\sigma_{M o t t}\left(v_{L} R_{L}+v_{T} R_{T}+v_{T T} R_{T T}+v_{T L} R_{T L}\right) \delta\left(M_{D}+\omega-E_{p}-E_{n}\right) d \varepsilon^{\prime} d \Omega_{e}^{\prime} d^{3} p^{\prime} .
$$

This is still a general expression, except that current conservation of the hadronic current is assumed.

All the quantities in Eq. (2.7) are determined in the lab frame. Considering the total four momentum of the np pair $P^{\prime}=\left(\mathbf{q}, E_{n p}\right)$ (with $\mathbf{q}=\mathbf{p}_{n}+\mathbf{p}_{p}$ and $E_{n p}=E_{n}+E_{p}$ ) an invariant mass $M_{n p}$ of the pair can be defined as $E_{n p}=\sqrt{\mathbf{q}^{2}+M_{n p}^{2}}$. Since the final state interaction of the final np pair can most readily be determined in its c.m. system $\left(P^{\prime c m}=\left(\mathbf{0}, M_{n p}\right)\right)$, we prefer to express Eq. (2.7) in terms of the structure functions $R_{\alpha}^{c m}$, evaluated in this frame. To make a distinction between lab and c.m. variables we use the convention that all variables in the c.m. frame are labeled by $\mathrm{cm}$. The relation can easily be found from

$$
J_{\mu}^{c m}=\mathcal{L}_{\mu}{ }^{\nu} J_{\nu}
$$

where $\mathcal{L}_{\mu}{ }^{\nu}$ describes the boost between the lab and c.m. frame. An explicit expression for the Lorentz transformation can be found using the relation $P_{\mu}^{\prime c m}=\mathcal{L}_{\mu}{ }^{\nu} P_{\nu}^{\prime}$ with

$$
\mathcal{L}_{\mu}{ }^{\nu}=\left(\begin{array}{cccc}
\sqrt{1+\eta} & 0 & 0 & -\sqrt{\eta} \\
0 & 1 & 0 & 0 \\
0 & 0 & 1 & 0 \\
-\sqrt{\eta} & 0 & 0 & \sqrt{1+\eta}
\end{array}\right)
$$

where we have

$$
\sqrt{\eta}=\frac{q}{M_{n p}}, \sqrt{1+\eta}=\frac{E_{n p}}{M_{n p}} .
$$

Since $\mathbf{q}$ is along the $\mathrm{z}$-axis, the transverse components of the deuteron current are not affected by the Lorentz boost. For the charge component we get $J_{0}^{c m}=\sqrt{1+\eta} J_{0}-\sqrt{\eta} J_{3}$. Together with current conservation $q \cdot J=q^{c m} \cdot J^{c m}=0$, we find a direct relation between the charge components of the current in the two frames 


$$
J_{0}=\frac{M_{n p}}{M_{D}} J_{0}^{c m} .
$$

We now turn to derive the differential cross section describing the exclusive $d\left(e, e^{\prime} p\right) n$ and inclusive $\left(e, e^{\prime}\right)$ reactions. In view of the Björken and Drell covariant normalization of the free np spinors, the transformation property of the $C_{n p}$ coefficient and the invariant volume element $d^{3} p^{\prime c m} / E_{p^{\prime}}^{c m}=d^{3} p^{\prime} / E_{p^{\prime}}$ we may rewrite Eq. (2.7) as

$$
d \sigma=\sigma_{M o t t}\left(v_{L} R_{L}+v_{T} R_{T}+v_{T T} R_{T T}+v_{T L} R_{T L}\right) \delta\left(E_{D}^{c m}+\omega^{c m}-2 E_{p^{\prime}}^{c m}\right) d \varepsilon^{\prime} d \Omega_{e}^{\prime} d^{3} p^{\prime c m},
$$

where the structure functions can be expressed in the corresponding observables in the c.m. system

$$
R_{L}=\left(\frac{M_{n p}}{M_{D}}\right)^{2} R_{L}^{c m}, R_{T}=R_{T}^{c m}, R_{T T}=R_{T T}^{c m}, R_{T L}=\left(\frac{M_{n p}}{M_{D}}\right) R_{T L}^{c m} .
$$

The exclusive reaction $d\left(e, e^{\prime} p\right) n$ is represented by a five fold differential cross section. In view of the invariant volume element we immediately find from Eq. (2.12)

$$
\frac{d^{5} \sigma}{d \varepsilon^{\prime} d \Omega_{e}^{\prime} d \Omega^{\prime}}=\sigma_{M o t t}\left(v_{L} \hat{R}_{L}+v_{T} \hat{R}_{T}+v_{T T} \hat{R}_{T T}+v_{T L} \hat{R}_{T L}\right),
$$

where we have absorbed the Jacobian $J$ into the definitions of the exclusive structure functions $\hat{R}_{\alpha} \equiv J R_{\alpha}$. For the Jacobian we have $J=\frac{d \Omega^{\prime c m}}{d \Omega^{\prime}} \frac{1}{2} p^{\prime c m} E_{p^{\prime}}^{c m}$ with

$$
\frac{d \Omega^{\prime c m}}{d \Omega^{\prime}}=\frac{p^{\prime}}{p^{\prime c m}} \frac{d E_{p^{\prime}}}{d E_{p^{\prime}}^{c m}}=\frac{p^{\prime}}{p^{\prime c m}} \frac{1}{\sqrt{1+\eta}}\left(1-\sqrt{\frac{\eta}{1+\eta}} \frac{E_{p^{\prime}}}{p^{\prime}} \cos \theta^{\prime}\right)^{-1}
$$

Hence, in principle the unpolarized exclusive reaction can be calculated by determining the four structure functions. For the $\left(e, e^{\prime}\right)$ process the integration over the momenta of the final np pair has to be performed. This is actually done in the c.m. frame of the np pair. Integration over the relative momentum $\mathbf{p}^{\prime c m}$ yields the inclusive $\left(e, e^{\prime}\right)$ cross section (in the lab frame)

$$
\frac{d^{3} \sigma}{d \varepsilon^{\prime} d \Omega_{e}^{\prime}}=\sigma_{M o t t}\left(v_{L} \bar{R}_{L}+v_{T} \bar{R}_{T}\right)
$$

where the inclusive structure functions $\bar{R}_{\alpha}$ are defined by

$$
\bar{R}_{\alpha}=2 \pi \int_{-1}^{1} R_{\alpha} \frac{1}{2} p^{\prime c m} E_{p^{\prime}}^{c m} d \cos \theta^{\prime c m}
$$

The momentum $p^{\prime c m}$ is determined by the energy conserving $\delta$ function in Eq. 2.12), i.e. from $E_{p^{\prime}}^{c m}=\frac{1}{2}\left(E_{D}^{c m}+\omega^{c m}\right)$. The contributions to the inclusive cross section from $R_{T T}$ and $R_{T L}$, which are proportional to $\cos 2 \phi^{\prime c m}$ and $\cos \phi^{\prime c m}$ respectively, vanish due to the $\phi^{\prime c m}$-integration. In section VI we will discuss this $\phi^{\prime c m}$ dependence in more detail.

\section{THE FORCE MODEL}

In determining the nuclear structure functions we in principle need to know the deuteron vertex function and the half off-shell nucleon-nucleon (NN) t-matrix. The nuclear interaction used by us is based on the one-boson-exchange (OBE) model. Following Fleischer and Tjon [6] it is assumed to be described by the exchange of $\pi, \rho, \omega, \eta, \varepsilon$ (or $\sigma$ ) and

$\delta$ mesons. A strong meson-nucleon form factor of the monopole type $F\left(p^{2}\right)=\frac{\Lambda^{2}}{\Lambda^{2}-p^{2}}$ is used to regularize the large momentum behavior. Within the relativistic field theory two-particle scattering can be described by the scattering t-matrix $\phi$, which satisfies the Bethe-Salpeter equation. The inhomogeneous Bethe-Salpeter equation for the t-matrix has the form

$$
\begin{aligned}
\phi\left(p^{\prime}, p ; P\right) & =V\left(p^{\prime}, p\right)-\frac{i}{4 \pi^{3}} \int d^{4} k \phi\left(p^{\prime}, k ; P\right) S_{2}(k, P) V(k, p) \\
& =V\left(p^{\prime}, p\right)-\frac{i}{4 \pi^{3}} \int d^{4} k V\left(p^{\prime}, k\right) S_{2}(k, P) \phi(k, p ; P),
\end{aligned}
$$


where

$$
\begin{aligned}
S_{2}(p, P) & =S^{(1)}(p, P) S^{(2)}(p, P) \\
& =\left(\frac{1}{2} \not P+\not p+M_{N}\right)^{(1)}\left(\frac{1}{2} \not P-\not p+M_{N}\right)^{(2)} G_{0},
\end{aligned}
$$

with $G_{0}=\left[\left(\frac{1}{2} P+p\right)^{2}-M_{N}^{2}+i \varepsilon\right]^{-1}\left[\left(\frac{1}{2} P-p\right)^{2}-M_{N}^{2}+i \varepsilon\right]^{-1}$. In Eq. (3.2) $S^{(n)}(p, P)$ is the free propagator of the n-th nucleon. The t-matrix also determines the deuteron vertex function $\Phi_{D}^{(M)}(\mathrm{p}, \mathrm{P})$ (where $\mathrm{M}$ is the polarization of the deuteron), corresponding to the residue at the deuteron bound state pole at $P^{2}=M_{D}^{2}$. For $P^{2} \approx M_{D}^{2}$ we have

$$
\phi\left(p^{\prime}, p ; P\right)=\sum_{M} \frac{\Phi_{D}^{(M)}\left(p^{\prime}, P\right) \tilde{\Phi}_{D}^{(M)}(p, P)}{P^{2}-M_{D}^{2}}+\text { regular terms. }
$$

Alternatively, $\Phi_{D}$ satisfies the homogeneous equation

$$
\Phi_{D}^{(M)}(p, P)=-\frac{i}{4 \pi^{3}} \int d^{4} k V(p, k) S_{2}(k, P) \Phi_{D}^{(M)}(k, P) .
$$

Although a field theoretical Bethe-Salpeter analysis is in principle possible for the case that $V$ is given by the OBE model, calculations are highly non trivial because of its analytic structure and computational complexity. To simplify this we use a relativistic quasipotential approximation. In the quasipotential framework the two-particle propagator is replaced by one where the relative energy variable is constrained (for a review see 15]). Here we use the choice which treats the two nucleons in a symmetric way $[9,10]$. It is given by

$$
G_{0} \rightarrow i \pi \delta\left(p_{0}\right) G_{2}^{B S L T}=i \pi \delta\left(p_{0}\right) \frac{1}{E_{p}-E} \frac{1}{\left(E_{p}+E\right)^{2}},
$$

where $E=\frac{1}{2} P_{0}$ and $E_{p}=\sqrt{M_{N}^{2}+\mathbf{p}^{2}}$. In this approximation the full two-particle propagator $S_{2}^{B S L T}$, containing the spinor structure (see Appendix A), is given by

$$
S_{2}^{B S L T}=\left(\frac{1}{2} \not p+\not p+M_{N}\right)^{(1)}\left(\frac{1}{2} \not p-\not p+M_{N}\right)^{(2)} G_{2}^{B S L T}=\left(E_{p}-E\right) S^{(1)}(p, P) S^{(2)}(p, P) .
$$

In this approximation the inhomogeneous Bethe-Salpeter equation (3.1) simplifies to

$$
\phi\left(\hat{p}, \hat{p}^{\prime} ; P\right)=V\left(\hat{p}, \hat{p}^{\prime}\right)+\frac{1}{4 \pi^{2}} \int d^{3} k \phi(\hat{p}, \hat{k} ; P) S_{2}^{B S L T}(\hat{k}, P) V\left(\hat{k}, \hat{p}^{\prime}\right),
$$

where $\hat{p}, \hat{p}^{\prime}$ and $\hat{k}$ are the four vectors $p, p^{\prime}$ and $k$, subject to the condition that their fourth component vanishes in the two-nucleon c.m. frame. To reconstruct the off-shell scattering wave function needed in the study of FSI effects a partial wave representation is used for the full NN amplitude. Following Ref. [6], the helicity basis is used. The representation is briefly discussed in Appendix A. The on-shell amplitude is simply obtained by taking $p=p^{\prime}$ with $\sqrt{P^{2}} \equiv \sqrt{s}=2 E_{p}$. Eq. (3.7) can be solved in a partial wave analysis. For details we refer to [6]. Because the quasipotential approximation is used, Eq. (3.7) reduces essentially to a coupled set of one-dimensional integral equations. Besides the physical $(+,+)$ positive energy states, also $(-,-)$ states and even and odd combinations of the $(+,-)$ energy states occur $[14]$.

Starting from the original fit of Ref. [6] (set A) for only positive energy spinor states, the meson-nucleon coupling constants $g_{\epsilon}$ and $g_{\delta}$ were adapted to the case that all the spinor states are included to get a reasonable fit to the experimental phase shifts of Arndt et al [16]. The resulting phase shifts up to $J=2$ are shown in Fig. 11 (set B), where the spectroscopic notation ${ }^{2 S+1} L_{J}$ has been used. For comparison the results of fit A are also plotted. To see what the effects are of the negative energy spinor states, we have switched these states off, keeping the same strength for the coupling constants. The results are shown as the dotted-dashed lines. Finally, an attempt was made to vary the meson coupling constants for the case of only positive energy states to reproduce the phase shifts as obtained with all spinor states included. We indeed obtain phase shifts (set C) which are not distinguishable from the set B in fig. 1. The sets of coupling parameters are given in Table I. 


\section{IMPULSE APPROXIMATION}

In order to construct a quasipotential approximation for inelastic electron scattering we start with the current operator as given in the Bethe-Salpeter formalism. The contributions to the current operator in the impulse approximation (IA) are given by the Feynman graphs shown in Fig. 2 and Fig. 3 in the case of inelastic electron scattering. In the contributions (a) and (b) ((c) and (d)) in Fig. 2 the photon couples directly to the proton (neutron) which is knocked out without any interaction with the other nucleon. These contribution are called the plane wave impulse approximation (PWIA) and the Born term respectively. The set of graphs in Fig. 3 corresponds to the contributions, where the outgoing nucleons interact after the virtual photon has been absorbed. These are the so-called final state interaction (FSI) contributions. In our calculations we will absorp the Born contribution in the FSI contributions. As is seen from the figure the FSI is expressed in terms of the half-off-shell NN t-matrix $\phi$. It satisfies the inhomogeneous equation given in Eq. (3.1). Including the Fermi character of the nucleons, the free np pair with total momentum $P^{\prime}$ and relative momentum $p_{f}$ is given by the antisymmetric combination of two free Dirac spinors with helicities $\lambda_{n}$

$$
<p_{f}, P^{\prime} \mid=\bar{u}_{\lambda_{1}}\left(\frac{1}{2} \mathbf{P}^{\prime}+\mathbf{p}_{f}\right) \bar{u}_{\lambda_{2}}\left(\frac{1}{2} \mathbf{P}^{\prime}-\mathbf{p}_{f}\right) A
$$

where A is the antisymmetrizer. As mentioned before we use the Kubis spinors. Since the outgoing particles are on-shell, we have to satisfy the two conditions

$$
p_{f} \cdot P^{\prime}=0, P_{0}^{\prime}=E_{\frac{1}{2} \mathbf{P}^{\prime}+\mathbf{p}_{f}}+E_{\frac{1}{2} \mathbf{P}^{\prime}-\mathbf{p}_{f}} .
$$

The PWIA and FSI contributions can be combined by introducing the scattering wave function for the two nucleons

$$
\tilde{\Psi}_{n p}\left(p_{f}, p^{\prime} ; P^{\prime}\right)=<p_{f}, P^{\prime} \mid\left[4 \pi^{3} i \delta^{4}\left(p^{\prime}-p_{f}\right)+\tilde{\phi}_{n p}\left(p_{f}, p^{\prime} ; P^{\prime}\right) S_{2}\left(p^{\prime}, P^{\prime}\right)\right]
$$

where $\tilde{\phi}_{n p}$ satisfies Eq. (3.1), subject to the condition that both outgoing particles are on mass shell. As a result, the current operator in the IA as represented in Figs. 2 and 3 can be written in the form

$$
J_{\mu}^{I A}=\frac{i e}{(2 \pi)^{4} M_{D}} \sum_{i=1}^{2} \int d^{4} p^{\prime} \tilde{\Psi}_{n p}\left(p_{f}, p^{\prime} ; P^{\prime}\right) \Gamma_{\mu}^{(i)}(q) S^{(i)}\left(p^{(i)}, P\right) \Phi_{D}\left(p^{(i)}, P\right),
$$

where $p^{(1)}=p^{\prime}-\frac{1}{2} q$ and $p^{(2)}=p^{\prime}+\frac{1}{2} q$. Because of four momentum conservation we have $P^{\prime}=P+q$. For the $\gamma N N$ vertex for the $\mathrm{k}$-th nucleon we take

$$
\Gamma_{\mu}^{(k)}(q)=F_{1}^{(k)}(q) \gamma_{\mu}^{(k)}+\frac{i}{2 M_{N}} F_{2}^{(k)}(q) \sigma_{\mu \nu}^{(k)} q^{\nu}
$$

with $F_{n}^{(k)}=F_{n}^{S}+\tau_{3}^{(k)} F_{n}^{V}$. In the actual studies we assume that the e.m. nucleon form factors $F_{n}$ can be described by their on-shell form. To obtain a similar form for the current matrix element as in the elastic case we may introduce the corresponding scattering vertex function

$$
\tilde{\Phi}_{n p}\left(p, p^{\prime} ; P\right)=\tilde{\Psi}_{n p}\left(p, p^{\prime} ; P\right) S_{2}^{-1}\left(p^{\prime}, P\right) .
$$

From studies of the two-nucleon functions $\Phi_{n p}$ and $\Phi_{D}$ 17 a rather smooth behavior is found in the relative energy $p_{0}$ variable. Therefore we assume that an expansion of the vertex functions in Eq. (4.4) around their relative variable $p_{0}=0$ point is reasonable in practical calculations. Keeping only the lowest order contribution and assuming that the resulting $\Phi^{\prime} s$ can be taken to be the BSLT vertex function, we get for the e.m. current operator

$$
J_{\mu}^{I A}=\frac{i e}{(2 \pi)^{4} M_{D}} \sum_{i=1}^{2} \int d^{4} p^{\prime} \tilde{\Phi}_{n p}^{B S L T}\left(\hat{p}^{\prime} ; P^{\prime}\right) S_{2}\left(p^{\prime}, P^{\prime}\right) \Gamma_{\mu}^{(i)} S^{(i)}\left(p^{(i)}, P\right) \Phi_{D}^{B S L T}\left(\hat{p}^{(i)} ; P\right) .
$$

Since the only $p_{0}$ dependence is in the nucleon propagators we may carry out the $p_{0}$ integration analytically. In Appendix B the explicit formulae of the propagator structure are given. The above approximation is essentially an equal time (ET) approximation to the current operator, i.e. with zero relative time.

Let us consider the covariance aspects of the quasipotential description. Under a given Lorentz transformation $\mathcal{L}$ we have 


$$
\begin{aligned}
V(k, p) & =\Lambda(\mathcal{L}) V\left(\mathcal{L}^{-1} k, \mathcal{L}^{-1} p\right) \Lambda^{-1}(\mathcal{L}), \\
S^{(i)}(p, P) & =\Lambda^{(i)}(\mathcal{L}) S^{(i)}\left(\mathcal{L}^{-1} p, \mathcal{L}^{-1} P\right) \Lambda^{(i)^{-1}}(\mathcal{L}), \\
\Phi_{D}(p, P) & =\Lambda(\mathcal{L}) \Phi_{D}\left(\mathcal{L}^{-1} p, \mathcal{L}^{-1} P\right), \\
\phi_{n p}(k, p, P) & =\Lambda(\mathcal{L}) \phi_{n p}\left(\mathcal{L}^{-1} k, \mathcal{L}^{-1} p, \mathcal{L}^{-1} P\right) \Lambda^{-1}(\mathcal{L}),
\end{aligned}
$$

with $\Lambda(\mathcal{L})=\Lambda^{(1)}(\mathcal{L}) \Lambda^{(2)}(\mathcal{L})$, where $\Lambda^{(i)}(\mathcal{L})$ is the corresponding boost operator in the Dirac space of the i-th nucleon. Because the BSLT deuteron and continuum wave functions transform in a similar way as the Bethe-Salpeter ones, the transformation property of the deuteron current for the quasipotential case is the same as that of the relativistic field theory case. Since

$$
\Lambda^{(1)^{-1}}(\mathcal{L}) \Gamma_{\mu}^{(1)}(q) \Lambda^{(1)}(\mathcal{L})=\mathcal{L}_{\mu}^{\nu} \Gamma_{\nu}^{(1)}\left(\mathcal{L}^{-1} q\right),
$$

we may conclude that the deuteron current given by Eq. (4.7) indeed transform as a four vector.

We close this section with some remarks on current conservation. As noted previously, for elastic electron deuteron scattering the current operator as defined in the Bethe-Salpeter formalism is conserved, provided the kernel of the Bethe-Salpeter equation satisfies a local property. One of the crucial ingredients in showing this is that the $\gamma N N$ vertex satisfies the Ward-Takahashi identity

$$
q \cdot \Gamma^{(i)}=F_{1}^{(i)}\left(S^{(i)^{-1}}\left(p^{(i)}, P^{\prime}\right)-S^{(i)^{-1}}(p, P)\right) .
$$

The analysis can readily be extended to the case of inelastic electron scattering. Note that in view of Eq. (3.1) the scattering wave function Eq. (4.3) satisfies the homogeneous equation

$$
\tilde{\Psi}_{n p}\left(p_{f}, p^{\prime} ; P^{\prime}\right) S_{2}^{-1}\left(p^{\prime}, P^{\prime}\right)=\frac{-i}{4 \pi^{3}} \int d^{4} k \tilde{\Psi}_{n p}\left(p_{f}, k ; P^{\prime}\right) V\left(k, p^{\prime}\right) .
$$

Substituting the Ward-Takahashi identity in Eq. (4.4) and using Eqs. (3.4) and (4.11) leads in the same way as in the elastic case to

$$
q \cdot J^{I A}=\frac{2 e}{(2 \pi)^{7} M_{D}} \sum_{i=1}^{2} \int d^{4} k \int d^{4} p \tilde{\Psi}_{n p}\left(k, P^{\prime}\right)\left[V\left(k, p^{i}\right), F_{1}^{(i)}\right] S_{2}(p, P) \Phi_{D}(p, P) .
$$

The main difference with the case of elastic electron scattering is that the final two-nucleon state is different from the initial state and that the e.m. nucleon form factors in the commutator also contain in this case in addition to the isoscalar also an isovector part. If the final state is an $I=0$ state the form factors are isoscalar and the commutator vanishes as for elastic scattering. In breakup reactions however also the $I=1$ channel is present in the final state, yielding a non vanishing isovector contribution. Consequently MEC contributions should be added to Eq. (4.12) to make it divergenceless. These will be considered in detail within the quasipotential approach in a forthcoming paper.

\section{ELASTIC SCATTERING IN ET APPROXIMATION}

The elastic deuteron current has precisely the same form as Eq. (4.7) except that $\tilde{\Phi} \rightarrow \tilde{\Phi}_{D}$. It is worth noting that the deuteron current for elastic scattering is conserved in the ET approximation at the level of positive energy states. This can be seen as follows. Keeping only the positive energy state contributions we get from Eq. (4.7)

$$
\begin{aligned}
q \cdot J^{I A}= & \frac{-e}{64 \pi^{5} M_{D}} \int d^{3} k \int d^{3} p \tilde{\Phi}_{D}^{B S L T}\left(\hat{k} ; P^{\prime}\right) S_{2}^{B S L T}\left(\hat{k}, P^{\prime}\right) \\
& \times \sum_{i=1}^{2}\left[V\left(\hat{k}, \hat{p}^{i}\right), F_{1}^{(i)}\right] S_{2}^{B S L T}(\hat{p}, P) \Phi_{D}^{B S L T}(\hat{p} ; P),
\end{aligned}
$$

since $1 / i \pi \int d p_{0} S_{2}(p, P)$ is equal to $S_{2}^{B S L T}\left(\frac{1}{2} \hat{p}, P\right)$ for positive energy states. Because now only the isoscalar parts of the form factors $F_{1}^{(i)}$ contribute to the current matrix elements for elastic scattering and these parts commute with the potential V, we may indeed conclude that Eq. (5.1) is divergenceless. 
Since the normalization condition of the deuteron wave function is related to current conservation, we should expect the correct normalization only for positive energy states. The normalization condition for the BSLT vertex function is given by

$$
P_{\mu} \delta_{M, M^{\prime}}=\frac{1}{(2 \pi)^{3}} \int d^{3} k \tilde{\Phi}_{D}^{(M)}(k ; P)\left(\frac{\partial}{\partial P_{\mu}} S_{2}^{B S L T}(k, P)\right)_{P^{2}=M_{D}^{2}} \Phi_{D}^{\left(M^{\prime}\right)}(k ; P) .
$$

This can readily be verified to hold. Using the identity

$$
-\frac{\partial}{\partial P_{\mu}} S_{2}(p, P)=\frac{1}{2}\left[S^{(1)}(p, P) \gamma_{\mu}^{(1)} S_{2}(p, P)+S^{(2)}(p, P) \gamma_{\mu}^{(2)} S_{2}(p, P)\right],
$$

we get for the ET current matrix element Eq. (4.7) in the limit $q \rightarrow 0$

$$
J_{\mu}^{I A}=\frac{-2 i e}{(2 \pi)^{4} M_{D}} \int d^{4} p \tilde{\Phi}_{D}^{B S L T}(\hat{p}, P) \frac{\partial}{\partial P_{\mu}} S_{2}(p, P) \Phi_{D}^{B S L T}(\hat{p}, P),
$$

which is indeed identical to Eq. (5.2) provided that only the positive energy states are kept. It should be noted that current conservation for the more general case can in principle be restored by adding an effective two-body current to Eq. (5.1) to cancel the four divergence of the IA current matrix element. Because the violation is at the level of the negative energy state contributions, the correction is of relativistic order.

The deuteron current (4.7) as discussed in the previous section is obviously different from the version studied in [8], where the BSLT two-body propagators were used for both the initial and final state propagators. Here we have kept the propagator structure and the $p_{0}$-integration is performed only over the propagators, since we have implicitly assumed that the $p_{0}$-dependence may be neglected in the vertex functions. We now compare the elastic electron scattering results as obtained with the two approaches. The calculations in the ET approach proceed in exactly the same way as in 8,18. Using the helicity framework (see Appendix A) we get for the current matrix element

$$
\begin{aligned}
<P^{\prime}, M^{\prime}\left|J_{\mu}^{I A}\right| P, M>= & \frac{2 i e}{(2 \pi)^{4} M_{D}} \int d k_{0} \int d k k^{2} \int d \Omega_{k} \\
& \times \sum_{n n^{\prime}} \sum_{m, m^{\prime}} \sum_{\tilde{\rho}, \tilde{\rho}^{\prime}} \tilde{\phi}_{n^{\prime}, m^{\prime}}^{B L T}\left(\mathbf{k}^{\prime}\right) S_{\rho^{\prime} \tilde{\rho}^{\prime}}^{(1)}\left(k^{\prime}\right) \tilde{\Gamma}_{\tilde{m}^{\prime}, \tilde{m}}^{(1)} S_{2_{\tilde{\rho} \rho}}(k) \phi_{n, m}^{B S L T}(\mathbf{k}),
\end{aligned}
$$

where we use the notation $m=\left\{\lambda_{1}, \lambda_{2}, \rho\right\} \tilde{m}=\left\{\lambda_{1}, \lambda_{2}, \tilde{\rho}\right\}$, with $\lambda_{i}$ being the helicity of the i-th nucleon and $\rho$ the quantum numbers of the rho-spin of the two-nucleon state. The angular quantum numbers are characterized by $n=\{J, M, L, S\}$.

The current matrix elements are evaluated in the Breit frame. Both initial and final states are boosted to their c.m. frame. On the $\rho$-spin basis $|++>|-,->, \mid e>$ and $\mid o>$ both propagators in Eq. (5.5) can be written in matrix form

$$
\left(\begin{array}{cccc}
S_{++} & 0 & 0 & 0 \\
0 & S_{--} & 0 & 0 \\
0 & 0 & S_{e e} & S_{e o} \\
0 & 0 & S_{o e} & S_{o o}
\end{array}\right)
$$

For the single nucleon propagator we have $S_{++}=S_{+}^{(1)}\left(k^{\prime}\right), S_{--}=S_{-}^{(1)}\left(k^{\prime}\right), S_{e e}=S_{o o}=\left(S_{+}^{(1)}\left(k^{\prime}\right)+S_{-}^{(1)}\left(k^{\prime}\right)\right) / 2$ and $S_{e o}=S_{o e}=\left(S_{+}^{(1)}\left(k^{\prime}\right)-S_{-}^{(1)}\left(k^{\prime}\right)\right) / 2$. The two-nucleon Green function $S_{2 \tilde{\rho} \rho}(k)$ can also be written in the form $(5.6)$ with $S_{++}=S_{2_{++}}(k), S_{--}=S_{2_{--}}(k), S_{e e}=S_{o o}=\left(S_{2_{+-}}(k)+S_{2_{-+}}(k)\right) / 2$ and $S_{e o}=S_{o e}=\left(S_{2_{+-}}(k)-S_{2_{-+}}(k)\right) / 2$. For the explicit form of these propagators we refer to Appendix A. We note that the matrices are no longer diagonal as is the case for the choice made in 8$]$ where the BSLT propagators are used.

In Fig. 4 are shown the results for the electric form factors $F_{C}$ and $F_{Q}$. The dashed line and the dotted-dashed line give respectively the results when all components or only positive energy states in the deuteron vertex function are included. We see that only at high momentum transfer the negative energy components contribute noticeably to the form factors. For comparison also are shown the results for the BSLT reduction as discussed in [8]. In both calculations the Höhler et al. [19] $\gamma N N$ form factors have been used. We see that the dip in the charge form factor $F_{C}$ is sensitive to the model used. At low momentum transfer the models give results in good agreement with each other. Only at high $q^{2}$ the results differ. In Fig. 5 are displayed the results for the magnetic form factor $F_{M}$. Negative 
energy components in the wave function yield large contributions to the form factor in the dip region. When these components are included, the position of the dip is shifted to higher momentum transfer. The two quasipotential reductions give different results when only positive energy states are taken into account. However, inclusion of the negative energy states results in a good agreement between the two approximations. The results for the electric form factor A and the magnetic form factor B are shown in Fig. 6 using the ET approximation. In the calculations of the $(\rho \pi \gamma$ and $\omega \varepsilon \gamma)$ MEC the BSLT propagator has been used instead of the ET one. They only differ (see Appendix A) for negative energy states. Calculations in various kinematic situations show that such a replacement doesnot affect the MEC results noticeably. The complete ET calculations of A yield essentially the same results as those with the BSLT approximation, except for some small differences at high momentum transfer. For the magnetic form factor the results deviate only in the dip region. The dip position in the ET approximation is at too low momentum transfer as compared to the data. Since the dip region is sensitive to the MEC coupling constants, no specific conclusions can be drawn from this. In particular, adapting the unknown $\omega \varepsilon \gamma$ coupling constant one can readily reproduce the experimental data. Also the tensor polarization $t_{20}$ are similar in both calculations. They agree with the experimental data (Fig. 7). At $q>4 \mathrm{fm}^{-1}$ the predictions of the ET approximation are somewhat above the results of the BSLT approximation, which is due to a lower value of the dip in the charge form factor.

\section{ELECTROMAGNETIC BREAKUP OF THE DEUTERON}

We now turn to describe the procedure to calculate the structure functions for inelastic electron scattering. There are two terms which are calculated separately. One is the PWIA and the other one is the FSI contribution. To determine the current matrix element we need to know the isospin structure. For the free final state we have both isospin $I=0$ or $I=1$, whereas the deuteron is an isospin $I=0$ state. Clearly, the isospin has to be chosen for a given total spin $S^{\prime}$ of the two-nucleon system in accordance with the final state being totally antisymmetric. Since the outgoing nucleons are on-mass shell we have the conditions $P_{0}^{\prime}=E_{\frac{1}{2} \mathbf{P}^{\prime}+\mathbf{p}_{f}}+E_{\frac{1}{2} \mathbf{P}^{\prime}-\mathbf{p}_{f}}$ and $p_{f} \cdot P^{\prime}=0$. In the following we consider the e.m. current matrix element with the final np pair in a total spin $\left(S^{\prime}, M_{S}^{\prime}\right)$ state.

\section{A. The PWIA contribution}

The simplest contribution to the deuteron current is the coupling of the photon to one of the two nucleons in the deuteron without FSI between the two outgoing nucleons. In Fig. 2 all possible contributions are depicted. Both initial and final states are antisymmetric and therefore the contributions (a) and (b) are identical. Let us now adopt the convention, where particle 1 in the final state is assumed to be the proton, carrying momentum $\frac{1}{2} P^{\prime}+p_{f}$. To determine the structure functions (2.13) we calculate the e.m. current matrix elements in the c.m. frame of the final state. In this frame we have $P_{0}^{\prime}=2 E_{p f}$ and $p_{f 0}=0$. The PWIA contribution can be written as

$$
\begin{aligned}
<P^{\prime}, p_{f}, S^{\prime}, M_{S}^{\prime}\left|J_{\mu}^{P W I A}\right| & P, M>=\frac{i e \sqrt{2}}{4 \pi M_{D}} \sum_{i=1}^{2} \\
\times & <P^{\prime}, p_{f}, S^{\prime}, M_{S}^{\prime} \mid \Gamma_{\mu}^{(i)} S^{(i)}\left(p^{(i)}, P\right) \Phi_{D}^{(M)}\left(p^{(i)}, P\right),
\end{aligned}
$$

where $p^{(1)}=p_{f}-\frac{1}{2} q$, and $p^{(2)}=p_{f}+\frac{1}{2} q$. The state $\mid P^{\prime}, p_{f}, S^{\prime}, M_{S}^{\prime}>$ describes the free np pair with total spin $S^{\prime}$ and its explicit form is given in Appendix A.

Since the deuteron vertex function is usually calculated in its rest system, it has to be boosted to the c.m. system of the final np pair. The Lorentz transformation which does this is given in Eq. (2.9). The corresponding one for spin- $\frac{1}{2}$ particles is given by

$$
\Lambda(\mathcal{L})=\sqrt{\frac{E_{D}+M_{D}}{2 M_{D}}}\left[1+\gamma^{0} \gamma^{3} \frac{-q}{E_{D}+M_{D}}\right] .
$$

Boosting the initial state to the c.m. frame of the np pair, the deuteron current contribution Eq. (6.1) can be written in the form

$$
\begin{aligned}
&<P^{\prime}, p_{f}, S^{\prime}, M_{S}^{\prime}\left|J_{\mu}^{P W I A}\right| P, M>=\frac{i e \sqrt{2}}{4 \pi M_{D}} \sum_{i=1}^{2} \\
& \times<P^{\prime}, p_{f}, S^{\prime}, M_{S}^{\prime} \mid \tilde{\Gamma}_{\mu}^{(i)} S^{(i)}\left(k^{(i)}, P\right) \Phi_{D}^{(M)}\left(k^{(i)}, P\right),
\end{aligned}
$$


where $\tilde{\Gamma}_{\mu}^{(i)}=\Gamma_{\mu}^{(i)} \Lambda(\mathcal{L}), \Lambda(\mathcal{L})=\Lambda^{(1)}(\mathcal{L}) \Lambda^{(2)}(\mathcal{L})$ and $k^{(i)}=\mathcal{L} p^{(i)}$

Introducing the deuteron state components $\phi_{n, m}\left(\mathbf{p}, p_{0}\right)$ labeled by $n=\{J, M, L, S\}$ and a combined helicity and $\rho$-spin label $m=\left\{\lambda_{1}, \lambda_{2}, \rho\right\}$, the deuteron current Eq. (6.3) can now be evaluated in the same way as has been done in the elastic case. For a more detailed discussion we refer to [8]. On the chosen basis the deuteron current is found to be of the form

$$
\begin{aligned}
<P^{\prime}, p_{f}, & S^{\prime}, M_{S}^{\prime}\left|J_{\mu}^{P W I A}\right| P, M>=\frac{i e \sqrt{2}}{4 \pi M_{D}} \sum_{i=1}^{2} \\
& \times \sum_{m^{\prime}} \sum_{\tilde{\rho} m, n} D_{M_{S}^{\prime} \lambda^{\prime \prime}}^{S^{\prime}}\left(\Omega_{f}\right) C_{\lambda_{1}^{\prime \prime}-\lambda_{2}^{\prime \prime} \lambda^{\prime \prime}}^{\frac{1}{2} \frac{1}{\prime} S^{\prime}} \tilde{\Gamma}_{\mu, m^{\prime}, \tilde{m}}^{(i)} S_{\tilde{\rho} \rho}^{(i)}\left(k^{(i)}, P\right) \phi_{n, m}\left(\mathbf{k}^{(i)}, k_{0}^{(i)}\right),
\end{aligned}
$$

where $m^{\prime}=\left\{\lambda_{1}^{\prime}, \lambda_{2}^{\prime},++\right\}$ and the explicit form of the one-particle propagator $S_{\tilde{\rho} \rho}^{(i)}$ is given in Appendix A and Eq. (5.6). The momentum $k^{(i)}$ is restricted by momentum conservation, since the spectator particle is on-shell we get that $k_{0}^{(1)}=E-E_{\vec{k}^{(1)}}$ and $k_{0}^{(2)}=-E+E_{\vec{k}^{(2)}}$. In writing down Eq. (6.4) we have shifted a $\gamma_{0}$ matrix to the vertex, so that the vertex operator is now given by $\tilde{\Gamma}_{\mu}^{(1)}=\Gamma_{\mu}^{(1)} \Lambda(\mathcal{L}) \gamma_{0}^{(2)}$ and $\tilde{\Gamma}_{\mu}^{(2)}=\Gamma_{\mu}^{(2)} \Lambda(\mathcal{L}) \gamma_{0}^{(1)}$. The extra $\gamma_{0}$ factor is due to the use of the closure relation for the helicity spinors. In Eq. (6.4) the $\varphi$-dependence can explicitly be evaluated. For the spherical components $\Gamma_{\mu}$ (with $\mu=0, \pm 1$ ) of the e.m. current we find that the total $\varphi$-dependence is given by $\exp \left[i\left(M+\mu-M_{S}^{\prime}\right) \varphi\right]$. With this $\varphi$-dependence of the current operator, $R_{L}$ and $R_{T}$ don't depend on $\varphi$ and is the $\varphi$-dependence of $R_{T L}$ and $R_{T T}$ given respectively by $\cos \varphi$ and $\cos 2 \varphi$. The isospin structure contained in $\Gamma_{\mu}$ can readily be evaluated. Since particle 1 is assumed to be the proton we get $\Gamma_{\mu}^{(1)} \rightarrow F_{p} / \sqrt{2}$ and $\Gamma_{\mu}^{(2)} \rightarrow F_{n} / \sqrt{2}$. In the actual calculations it is convenient to treat the proton and neutron contributions to the e.m. current separately. Denoting the term where the photon interacts with the proton (neutron) as $J_{\mu}^{(p)}\left(J_{\mu}^{(n)}\right)$ we find with the help of symmetry relations for the deuteron vertex functions (see [8])

$$
J_{\mu}^{(n)}\left(\mathbf{p}_{f}\right)=(-1)^{L+S+S^{\prime}} J_{\mu}^{(p)}\left(-\mathbf{p}_{f}\right)
$$

with all e.m. proton form factors in $J_{\mu}^{(p)}$ replaced by the corresponding ones of the neutron.

The ET approximation for the PWIA contribution can be obtained immediately from the above expressions, by simply replacing the initial deuteron bound state by the BSLT vertex function. The relative energy variable in the propagator is prescribed by the condition that the final state describes on-shell particles.

\section{B. Rescattering contribution}

When FSI is included in the IA contributions, the structure is more complicated. Starting from the full BetheSalpeter formalism the current matrix elements has the form given in Eq. (4.4). We have

$$
\begin{aligned}
<P^{\prime}, p_{f}, S^{\prime}, M_{S}^{\prime} & \left|J_{\mu}^{F S I}\right| P, M>=\frac{e}{(2 \pi)^{4} M_{D}} \sum_{i=1}^{2} \int d^{4} p^{\prime} \\
& \times \tilde{\phi}_{n p}\left(p_{f}, p^{\prime}, P^{\prime}\right) S_{2}\left(p^{\prime}, P^{\prime}\right) \Gamma_{\mu}^{(i)} S^{(i)}\left(p^{(i)}, P\right) \Phi_{D}^{(M)}\left(p^{(i)}, P\right) .
\end{aligned}
$$

Assuming again that particle 1 in the final state is the proton, we only have to take the two graphs (a) and (b) into account as represented in Fig. 3. Using the same analysis leading to Eq. (6.4) we find

$$
\begin{aligned}
& <P^{\prime}, p_{f}, S^{\prime}, M_{S}^{\prime}\left|J_{\mu}^{F S I}\right| P, M>=\frac{e}{(2 \pi)^{4} \sqrt{2} M_{D}} \sum_{i=1}^{2} \sum_{n^{\prime}, n} \sum_{m, m^{\prime}, m^{\prime \prime}} \sum_{\tilde{\rho} \tilde{\rho}^{\prime}} \int d^{4} p^{\prime} \\
& \times D_{M_{S}^{\prime} \lambda^{\prime}}^{S^{\prime}}\left(\Omega_{f}\right) C_{\lambda_{1}^{\prime}-\lambda_{2}^{\prime} \lambda^{\prime}}^{\frac{1}{2} \frac{1}{2} S^{\prime}} \tilde{\phi}_{n^{\prime} ; m^{\prime}, m^{\prime \prime}}\left(p_{f}, p^{\prime}\right) S_{2_{\rho^{\prime \prime} \tilde{\rho}^{\prime \prime}}} \tilde{\Gamma}_{\mu, \tilde{m}^{\prime \prime} \tilde{m}}^{(i)} S_{\tilde{\rho} \rho}^{(i)}\left(k^{(i)}, P\right) \phi_{n, m}\left(\mathbf{k}^{(i)}, k_{0}^{(i)}\right),
\end{aligned}
$$

where the partial wave components $\tilde{\phi}_{n^{\prime} ; m^{\prime}, m^{\prime \prime}}\left(p_{f}, p^{\prime}\right)$ correspond to the isospin dependent t-matrix elements with total angular momentum $n^{\prime}=\left\{J^{\prime}, M^{\prime}\right\}$ between the energy-helicity spin states $m^{\prime}=\left\{\lambda_{1}^{\prime}, \lambda_{2}^{\prime},++\right\}$ and $m^{\prime \prime}=\left\{\lambda_{1}^{\prime \prime}, \lambda_{2}^{\prime \prime}, \rho^{\prime \prime}\right\}$. The isospin dependence of $\tilde{\Gamma}$ in Eq. (6.7) is for an isoscalar transition given by $F_{S} / \sqrt{2}$ and for an isovector transition $(-1)^{i} F_{V} / \sqrt{2}$. 
Replacing the integration variable $p^{\prime}$ by $-p^{\prime}$ in the integral we readily see that both contributions are equal. Moreover, collecting all the $\varphi$-dependence we find $e^{i\left(M^{\prime}-M_{S}^{\prime}\right) \varphi_{f}} e^{i\left(M+\mu-M^{\prime}\right) \varphi^{\prime}}$, so that the $\varphi^{\prime}$-integration can be performed with the result

$$
2 \pi \delta\left(M+\mu-M^{\prime}\right) e^{i\left(M+\mu-M_{S}^{\prime}\right) \varphi_{f}} .
$$

Notice that the same $\varphi_{f}$-dependence exists as for the PWIA. In both the initial and final state the relative energy variable is set to zero, corresponding the BSLT prescription. Since the only $p_{0}^{\prime}$-dependence is in the nucleon propagators, the $p_{0}^{\prime}$-integration can be done analytical. As a result we are left with a two-dimensional integral which can be done numerically using standard gaussian quadratures. In so doing, one particular point has to be taken care of. At the point $E_{p^{\prime}}=\frac{1}{2} M_{n p}$ the particles are on mass-shell and we have a singularity in the positive energy propagator, which is of the form $1 /\left(E_{p^{\prime}}-\frac{1}{2} M_{n p}\right)$. This singularity can simply be dealt with using a standard subtraction technique.

Having determined the e.m. current matrix elements in this way the deuteron structure functions can be constructed using Eq. (2.6). It should be noted that not all partial wave matrix elements in Eq. (6.7) need explicitly to be calculated. Due to parity conservation we have

$$
<J^{\prime} M^{\prime} L^{\prime} S^{\prime}\left|J_{\mu}\right| P, M>=(-1)^{J+J^{\prime}+M+M^{\prime}+\mu}<J^{\prime}-M^{\prime} L^{\prime} S^{\prime}\left|J_{\mu}\right| P,-M>.
$$

Besides this symmetry relation we have the selection rule $M^{\prime}=M+\mu$ for the above matrix element, being a consequence of rotational symmetry.

\section{RELATIVISTIC ANALYSIS OF INELASTIC ELECTRON SCATTERING}

In the preceding sections we have developed a relativistic framework to describe electrodisintegration of the deuteron. The full relativistic form of the e.m. operator is employed and at the same time the relativistic structure of the nuclear dynamics is incorporated. To see what kind of predictions our fully relativistic formulation leads to in the case of inelastic electron scattering we study the structure functions for two kinematic situations. One is the inclusive experiment carried out by the Bates-Mit group and the other is the exclusive analysis by the Nikhef-K group. Since we also want to compare our predictions to those obtained from nonrelativistic calculations, let us first discuss the e.m. operator used in the Schrödinger approach.

\section{A. Nonrelativistic limit}

In most of the studies of electron-deuteron scattering it is implicitly assumed that to a good approximation the deuteron can be described by a boundstate wave function satisfying a Schrödinger equation. In addition some effective nonrelativistic (NR) form is used for the e.m. operator. Various versions have been proposed in the literature based on taking the NR limit of the current operator. The e.m. operator is expanded in $q / M$, keeping only the first order term in the expansion and neglecting recoil effects. Because NR wave functions are used, boost effects are in general also neglected. The resulting effective e.m. operators have all in common to be the same in leading order, but to differ in relativistic order $(q / M)^{2}$ Since we have included in our study the full relativistic structure of the e.m. operator, it is obviously of interest to see how these relativistic corrections look like. In the calculations the vertex is defined in the $\mathrm{cm}$-frame of the final state. Because the helicities in this frame and the $\mathrm{cm}$-frame of the initial deuteron state are not equal, we have to replace the boost operators by Wigner rotations. A detailed discussion is given in Ref. [8].

In the NR limit all particles are taken to be on-shell and the negative energy state components are neglected, i.e. since the nucleons are assumed to behave nonrelativistically, we may assume that the nucleons can be described by the positive energy spinors

$$
\begin{aligned}
& u_{\lambda}^{(1)}(\mathbf{p})=N_{p}\left[\begin{array}{c}
1 \\
\frac{\sigma \cdot \mathbf{p}}{E_{p}+M}
\end{array}\right] \chi_{\lambda}, \\
& u_{\lambda}^{(2)}(\mathbf{p})=N_{p}\left[\begin{array}{c}
1 \\
\frac{-\sigma \cdot \mathbf{p}}{E_{p}+M}
\end{array}\right] \chi_{-\lambda},
\end{aligned}
$$

where $N_{p}=\sqrt{E_{p}+M_{N} / 2 E_{p}}$ and $\chi_{\lambda}$ are the Pauli spinors with helicity $\lambda$. Therefore the current matrix element to study in this approximation is given by 


$$
J_{\mu}=\bar{u}_{\lambda^{\prime}}\left(\mathbf{p}^{\prime}\right)\left(F_{1} \gamma_{\mu}+\frac{i}{2 M_{N}} F_{2} \sigma_{\mu \nu} q^{\nu}\right) u_{\lambda}(\mathbf{p}) .
$$

It can immediately be reduced to a Pauli form. We get

$$
\begin{aligned}
J_{0}= & F_{1}-\frac{1}{8 M_{N}^{2}}\left(F_{1}+2 F_{2}\right)\left(\mathbf{q}^{2}+i \sigma \cdot[\mathbf{K} \times \mathbf{q}]\right)+O\left(\frac{q^{4}}{M_{N}^{4}}\right) \\
\mathbf{J}= & \frac{F_{1}}{2 M_{N}}\left[(\mathbf{K}+i[\sigma \times \mathbf{q}])\left(1-\frac{1}{8 M^{2}}\left\{\mathbf{K}^{2}+\mathbf{q}^{2}\right\}\right)-\frac{1}{8 M^{2}}(\mathbf{q}+i[\sigma \times \mathbf{K}])(\mathbf{K} \cdot \mathbf{q})\right] \\
& +\frac{F_{2}}{2 M_{N}}\left[i[\sigma \times \mathbf{q}]\left(1-\frac{1}{8 M^{2}} \mathbf{q}^{2}\right)-\frac{\omega}{2 M_{N}}(\mathbf{q}+i[\sigma \times \mathbf{K}])\right. \\
& \left.+\frac{1}{8 M^{2}}([\mathbf{K} \times \mathbf{q}] \times \mathbf{q}-i(\sigma \cdot \mathbf{K})[\mathbf{K} \times \mathbf{q}])\right]+O\left(\frac{q^{4}}{M_{N}^{4}}\right),
\end{aligned}
$$

with $\mathbf{K}=\mathbf{p}+\mathbf{p}^{\prime}$ and where we have kept all second order terms.

In the usual NR limit only the first order terms are kept in the charge and current operator. In so doing, we get the operator as used by Mathiot [25] and Sommer [26]

$$
J_{0}=F_{1}, \mathbf{J}=\frac{1}{2 M_{N}} F_{1} \mathbf{K}-\frac{i}{2 M_{N}} G_{M}(\mathbf{q} \times \sigma) .
$$

Note that the correction terms in Eq. (7.3) are a rather complicated function of both $q$ and $K$. Except for the region of low $q$ and small recoil corrections there is no a priori reason to expect them to be small.

Another form which is often used, is found by starting from the alternative form of the relativistic current operator in terms of Sachs form factors

$$
G_{E}=F_{1}+\frac{q^{2}}{4 M_{N}^{2}} F_{2}, G_{M}=F_{1}+F_{2}
$$

Taking the NR limit in this case yields the e.m. operator used for example by Leidemann and Arenhövel [27]

$$
J_{0}=G_{E}, \mathbf{J}=\frac{1}{2 M_{N}} G_{E} \mathbf{K}-\frac{i}{2 M_{N}} G_{M}(\mathbf{q} \times \sigma) .
$$

The only difference between the two approximations is the use of $F_{1}$ or $G_{E}$ in the charge and current operator. From Eq. (7.5) we see that the difference is of order $q^{2} / M_{N}^{2}$, being of relativistic origin. Considering Eq. (7.3) we see that the charge operator can be rewritten up to order $q^{4}$ as

$$
J_{0}=G_{E}\left(1-\frac{\mathbf{q}^{2}}{8 M_{N}^{2}}\right)-\frac{1}{8 M_{N}^{2}}\left(2 G_{M}-G_{E}\right) i \sigma \cdot(\mathbf{K} \times \mathbf{q}) .
$$

Momentum conservation at the vertex gives $\mathbf{K}=\mathbf{p}+\mathbf{p}^{\prime}=2 \mathbf{p}+\mathbf{q}$. Hence for small values of the missing momentum, i.e. $p<<q$, the second term in Eq. (7.7) can be neglected. For values of $q$ up to a few hundred $\mathrm{MeV}$ it suggests that the choice of $G_{E}$ is indeed reasonable for the charge operator. The procedure as followed in Eq. (7.7) can not be used for the current operator, because of the lack of a small parameter. However, we can show that the isovector part up to order $q^{4}$ is given by

$$
-\frac{i}{2 M_{N}} G_{M}\left(1-\frac{3 \mathbf{q}^{2}}{8 M_{N}^{2}}\right)(\mathbf{q} \times \sigma),
$$

which shows that the choice of $G_{M}$ in Eq. (7.4) and Eq. (7.6) is reasonable for small $q$. It should be noted that in some calculations of Ref. [27] the factor $C_{n p}=\frac{M_{N}^{2}}{E_{p} E_{n}}$ has been added to the cross section, whereas this term is already included in Eq. (7.3). This correction is clearly of relativistic order.

Besides the additional terms neglected for a given choice of the NR e.m. operator, other relativistic effects like contributions from the boost transformation and the negative energy state components of the deuteron and scattering wave function, can play an important role. In Fig. 8 are shown the relative contributions from these corrections to the response functions for an exclusive experiment with missing momentum $p_{m}=100 \mathrm{MeV} / \mathrm{c}$ and $q$ in the range of 300 
$\mathrm{MeV} / \mathrm{c}$ to $1500 \mathrm{MeV} / \mathrm{c}$. In addition we used $\theta_{p}=120^{\circ}$ and $\theta_{e}=60^{\circ}$ and took the kinematics in plane. In this figure we are comparing the different approximations to the complete relativistic calculation with FSI included, but without negative energy state contributions. The effect of including the negative energy state contributions is given by the double dot-dashed curve. As a second approximation we may drop the boost operators in the current operator (solid line). This is similar to the NR approximations except that we keep the complete current operator Eq. (7.2). If only the PWIA contribution is considered all response functions show identical dependence on the boost operators. The contributions from the boost to $R_{L}, R_{T}$ and $R_{T L}$ become more important at higher $q$ values and can be as large as $40 \%$ around $q=1500 \mathrm{MeV} / \mathrm{c}$, whereas in the case of $R_{T T}$ inclusion of the FSI substantially enlarges this dependence and the boost effects are large even for low momentum transfer. The negative energy state contributions increase at higher $\mathrm{q}$ and are of the order of $5 \%$ around $\mathrm{q}=300 \mathrm{MeV} / \mathrm{c}$. Again a different behavior is found for $R_{T T}$, an effect of around $30 \%$ is seen for the whole kinematic region. An important conclusion is that the negative contributions cancel to a large extent the boost effects. Similar as for elastic electron deuteron scattering a consistent relativistic treatment is also important in this case.

Next we may consider the effect of neglecting the FSI contributions. This yields the long dashed curve in Fig. 8. As expected we see an increase in the FSI contribution at lower q, since q is directly related to the energy of the np-pair in the final state. Also it is of some interest to see the sensitivity on the choice of the relativistic two-nucleon propagator on the response functions. The dashed line depicts the relative difference between the BSLT and ET approximation, including FSI. Except for $R_{T T}$, the difference is less than $5 \%$ and increases for higher values of q, whereas for $R_{T T}$ it decreases with q. This dependence on the propagator structure only exists if FSI is included.

Finally, also plotted in Fig. 8 are the predictions with the e.m. operators, given by Eq. (7.4) and Eq. (7.6) with the $F_{1}$ and $G_{E}$ form factors. The results are respectively given by the dotted and the dot-dashed lines. As expected we see increasing deviations with increasing q. For $R_{L}$ the NR $G_{E}$ operator yields clearly the closest resemblance to the calculation using the complete current operator. The latter is in accordance with our discussion of Eq. (7.7) that the use of $G_{E}$ in the charge operator should be reliable in the quasielastic region. Beause of isovector dominance in the contribution of $R_{T}$ both NR operators give the same results and deviate only a few percent of the results with the exact current operator, what is in accordance with Eq. (7.8). Finally we see from the figure that the deviations are very large for the response functions $R_{T L}$ and $R_{T T}$ even at comparatively low momentum transfer. These deviations are essentially due the interference structure of the response functions, therefore probing those parts of the current operators not described well by the NR operators.

Not shown in these figures are the results with the current operator of Eq. (7.3) where we have kept all second order terms. Similar higher order corrections have also been considered recently [28 29] For $R_{L}$ and $R_{T}$ although the results agree reasonably well with the full calculations at moderate momentum transfer they are found to be substantially different at higher momenta. In general at high momentum transfers the deviations are such that the complete e.m. operator including boost effects is needed for a reliable analysis of the response functions. For $R_{T T}$ and $R_{T L}$ calculations with this second order operator show it gives comparable results as with the complete operator. Deviations are within a few percent. At these low missing momenta no large model dependence of the wave functions is expected. The deuteron wave function is mainly $\mathrm{S}$-wave in this region. We have tested the aspect of the non relativistic reduction of the propagators. With this approximation we find effects smaller than $0.5 \%$.

To get some insight about sensitivities on FSI and negative energy states in a larger part of the phase space in Figs. 9 and 10 contour plots are shown where we have varied in addition $\theta_{p}$. For the relative contribution of the FSI, shown in Fig. 9, we find very similar effects in the various kinematic regions for $R_{L}$ and $R_{T}$. From these figures we see that both contributions to in particular the $R_{T T}$ response function can be substantial.

\section{B. Structure functions of the exclusive reaction}

As an example we discuss the exclusive experiments carried out at Nikhef-K 11.21. In these experiments in addition to the measured final electron also the outcoming proton is detected. As a result the cross section is described by the four response functions $R_{L}, R_{T}, R_{T L}$ and $R_{T T}$ given in Eq. (2.6). $R_{T L}$ and $R_{T T}$ have an out of plane dependence of respectively $\cos \phi$ and $\cos 2 \phi$. Consequently $R_{T L}$ can be separated by carrying out two measurements at $\phi=0$ and $\phi=\pi$, such that $\mathbf{p} \cdot \mathbf{q}$ is constant. The response function $R_{T L}$ is interesting because through its interference structure it is sensitive to the isovector part of the charge operator and the isoscalar part of the current operator. By measuring only $R_{L}$ and $R_{T}$ no information is obtained about these terms because of the dominance of the isoscalar charge and isovector current operators. The experiments were performed with a missing momentum $p_{m}$ in the range of 40 to $180 \mathrm{MeV} / \mathrm{c}$. In this kinematic region the FSI is expected to give only small contributions due to the low missing momentum and therefore the high relative momenta of the outcoming nucleon pair. Because of the dominance of the PWIA contribution the chosen kinematics is very suitable to examine the underlying structure of the one-body 
current operator.

In Fig. 11 the results of the Nikhef experiments together with our predictions are shown. In all these calculations the OBE model has been used to construct the continuum and deuteron vertex functions. In these figures PWIA corresponds to our relativistic PWIA calculation and the curve denoted by REL(-) contains in addition the FSI (both positive and negative energy states). For both $R_{L}$ and $R_{T}$ the FSI is small and increases with increasing missing momentum. Also are plotted the predictions obtained using a nonrelativistic description with e.m. operators, given by Eq. (7.4) and Eq. (7.6) with $F_{1}$ and $G_{E}$ form factors. The results show clearly that the current operator with $F_{1}$ is too high as compared to the experimental data for $R_{L}$ and the $G_{E}$ description gives a much better agreement with both the data and the relativistic calculation. The results for $R_{T L}$ and $R_{T T}$ are remarkable. Although we are at relatively low momentum transfer, relativistic effects are substantial. We find that our relativistic calculations leads to a good description of the $R_{T L}$ data, whereas both NR predictions are clearly too low. In this case the NR $F_{1}$ operator is closer to the relativistic prediction. Similar conclusions can be drawn for the out of plane structure function $R_{T T}$. It is worth noting that for this case the FSI effect is considerably larger.

From these results we may conclude that in the kinematic region considered the most important relativistic effects arise from the higher order corrections to the e.m. operators employed in the usual NR analysis. In general the NR descriptions are clearly not adequate and they can represent a poor approximation to the full relativistic operator.

\section{Inclusive scattering in the quasielastic region}

We now turn to describe our calculations for the inclusive reactions in the quasielastic region. These experiments were done at Mit-Bates for momentum transfers $q$ in the range of $300-500 \mathrm{MeV} / \mathrm{c}$ [5]. The quasielastic peak is defined by

$$
M_{D}+\omega=\sqrt{M_{N}^{2}+q^{2}}+M_{N}
$$

In the considered experiments a longitudinal-transverse separation has been carried out. The four momentum transfer is such that Eq. (7.9) to a good approximation reduces to $\omega=q^{2} / 2 M_{N}$. For these values of $\omega$ and $q$ the electron is essentially probing the nucleons with missing momentum near zero.

In Figs. 1214 are shown the various corrections to the longitudinal and transverse response functions at momentum transfer $\mathrm{q}=300,400$ and $500 \mathrm{MeV} / \mathrm{c}$. The full relativistic expression of the electromagnetic vertex is used. Inclusion of the boost operator shows only a very small contribution. Considering $R_{L}$ we see that the PWIA describes to a good approximation the response, except at lower momentum transfer. The Born contribution can essentially be neglected, because $F_{1}^{n}$ is very small. On the other hand the FSI is seen to be important for $R_{T}$ over the whole range of considered momentum transfer. Moreover, since the magnetic form factor of the nucleon is comparable in magnitude to $F_{2}^{n}$ the Born term also gives a substantial contribution to $R_{T}$. For larger $\omega$ the delta-degree of freedom is seen to come up. This contribution and the other MEC's will be discussed in the same relativistic framework in a next paper.

Including only respectively the Born, the $\mathrm{J}=0$ and $1 \mathrm{NN}$ scattering amplitude in the two response functions we see that most of the FSI has been accounted for . The main FSI contribution to $R_{L}$ is from the $J=1\left({ }^{1} P_{1},{ }^{3} S_{1}-{ }^{3} D_{1},{ }^{3} P_{1}\right)$ amplitudes. Especially at low $\omega$ this contribution can be significant due to the decreasing relative momentum of the outcoming nucleon pair. Similarly as for the three-nucleon systems 30 the response increases at low $\omega$, whereas it decreases at the quasielastic peak due to the FSI contribution. The PWIA result for $R_{T}$ is clearly below the data. In particular, the $J=0\left({ }^{1} S_{o},{ }^{3} P_{o}\right)$ FSI contribution is only of some importance for $R_{T}$. Due to the FSI contributions our overall predictions are well in accordance with the experimental data. Although substantially smaller than in the the trinucleon system some strength tends to be missing in the transverse response function at lower energy transfer. We also considered the negative energy state contributions. Exactly as we have found for exclusive scattering this contribution seems to be vanishing at the quasielastic peak. Furthermore it reduces the strength at low $\omega$, whereas it tends to increase slightly the response functions past the quasielastic peak.

The overall agreement with the experimental data is gratifying. Since at these relatively low momentum transfers the existing parameterizations of the e.m. nucleon form factors are very similar, less dependence is found on the choice of these nucleon form factors. Using for example the Iachello et al [31] form factors changes of only at most 1 percent were found.

\section{SUMMARY AND OUTLOOK}

We have presented in this paper a framework to study the e.m. deuteron current based on a relativistic quasipotential approach. The nuclear dynamics itself is described in terms of a symmetric choice for the relative energy variable. 
Assuming that the two-nucleon vertex functions depend smoothly on this variable, the current matrix element is determined by explicitly integrating out the relative energy variable in the e.m. vertex loop. This equal time approximation has the advantage that it can be used to describe both elastic and inelastic electron scattering and that it in principle can be extended in a systematic way. As a direct application the elastic e.m. properties of the deuteron are studied using this formalism. The differences between this approach and the one used in Ref. [8] for elastic electron deuteron scattering are the treatment of the initial and final states and the nucleon-propagator structure of the one-body current. We have shown that for elastic scattering these two approaches give compatible results and differences are found to be minor.

The approach discussed here has clearly the advantage that besides elastic electron scattering case, it can also be applied to the deuteron breakup in electro- and photo-disintegration processes. Explicit expressions are written down for the deuteron current in the case of inelastic electron scattering. The helicity formalism is extensively used to relate the scattering wave functions of the relativistic one boson exchange model to the current matrix element. Since there are also isovector current contributions in this case, mesonic exchange currents are in general needed to satisfy gauge invariance. These currents can also be constructed in this ET approximation and will be studied in a subsequent paper.

Various different forms of one-body operators exist in the literature to describe the e.m. processes in a nonrelativistic framework. These are derived from relativistic operators which are on-shell equivalent, but lead to different operators in the NR limit. We have compared the NR calculations with our relativistic description of the electro disintegration of the deuteron. The important difference is that we also take in our approach the higher order terms in $q / M$ into account. It should be noted that we have assumed a specific on shell form of the e.m. nucleon form factor and not considered its off-shell dependence. For this a detailed dynamical model of the nucleon is needed.

One interesting experiment to study the difference between a relativistic and nonrelativistic analysis is the exclusive experiment performed at Nikhef-K, in which the three response functions $R_{L}, R_{T}$ and $R_{T L}$ could be separated. Due to the small missing momentum in these experiments the FSI-contribution is very small and the response functions are in a good approximation described by the one-body current (PWIA). In our analysis we have found that both relativistic and nonrelativistic theories give a good description of $R_{T}$. Differences show up for $R_{L}$, the NR-theory using the $G_{E}$ form factor gives a good description, while the $F_{1}$ current operator is systematic to high. Interesting results were found for the interference structure function $R_{T L}$. Both NR-approaches failed to describe the data. Including the next order in $q / M$ to the one-body current operator showed a much better agreement. Other relativistic effects have also been considered. These are due to boost effects and the presence of negative energy states. In the kinematics studied these contributions were found to be small. However, it is expected that these corrections will be substantial at higher momentum and energy transfer as can be seen in the case of elastic electron scattering. We have tested this assumption by considering the range of $q=300 \mathrm{MeV} / \mathrm{c}$ to $1500 \mathrm{MeV} / \mathrm{c}$. Effects of $40 \%$ were found. The second experiment we discussed in our relativistic approach is the inclusive experiment done at Bates. In this experiment much larger effects due to the Born term and FSI are found. A good agreement with the data is found, although at larger momentum transfer $q$ the longitudinal response function is slightly overestimated. This may however readily be explained in terms of model dependence on the deuteron structure. For these experiments the NR-description using $G_{E}$ gives similar results as the relativistic calculation.

\section{APPENDIX A: HELICITY BASIS}

In this Appendix we summarize the helicity basis used in the partial wave decomposition of the NN amplitude. For more details we refer to Refs. [6, 14, 32]. Following Kubis [14] we use for the positive and negative energy spinors

$$
\begin{aligned}
& u_{\lambda}^{(+)}(\mathbf{p})=N_{p}\left[\begin{array}{c}
1 \\
\frac{2 \lambda p}{E_{p}+M}
\end{array}\right] \chi_{\lambda}(\theta, \varphi), \\
& u_{\lambda}^{(-)}(\mathbf{p})=N_{p}\left[\begin{array}{c}
\frac{-2 \lambda p}{E_{p}+M} \\
1
\end{array}\right] \chi_{\lambda}(\theta, \varphi),
\end{aligned}
$$

where $\chi_{\lambda}(\theta, \varphi)$ are the two-component Pauli spinors, which are given by

$$
\begin{aligned}
\chi_{\frac{1}{2}}(\theta, \varphi) & =\left(\begin{array}{c}
\cos \theta / 2 \\
e^{i \varphi} \sin \theta / 2
\end{array}\right), \\
\chi_{-\frac{1}{2}}(\theta, \varphi) & =\left(\begin{array}{c}
-e^{-i \varphi} \sin \theta / 2 \\
\cos \theta / 2
\end{array}\right) .
\end{aligned}
$$


These spinors are normalized as

$$
u_{\lambda}^{(\rho)^{\dagger}}(\mathbf{p}) u_{\lambda^{\prime}}^{\left(\rho^{\prime}\right)}(\mathbf{p})=\delta_{\rho \rho^{\prime}} \delta_{\lambda \lambda^{\prime}},
$$

where $\rho= \pm$ and the normalization is given by $N_{p}=\sqrt{\left(E_{p}+M_{N}\right) / 2 E_{p}}$. Let us consider the projection operators

$$
\Lambda_{\rho}(\mathbf{p})=\sum_{\lambda} u_{\lambda}^{(\rho)}(\mathbf{p}) \bar{u}_{\lambda}^{(\rho)}(\mathbf{p}) .
$$

We may rewrite the single nucleon propagator

$$
S(p)=\left(\not p-M_{N}+i \varepsilon\right)^{-1}=\frac{\not p+M_{N}}{p^{2}-M_{N}^{2}+i \varepsilon} .
$$

in terms of these projection operators. Using the relation

$$
\not p+M_{N}=\left(E_{p}+p_{0}\right) \Lambda_{+}(\mathbf{p})+\left(p_{0}-E_{p}\right) \Lambda_{-}(\mathbf{p})
$$

we get for the propagator

$$
S(p)=\frac{\Lambda_{+}(\mathbf{p})}{p_{0}-E_{p}+i \varepsilon}+\frac{\Lambda_{-}(\mathbf{p})}{p_{0}+E_{p}+i \varepsilon} .
$$

We now turn to discuss briefly the partial wave representation of the two-nucleon t-matrix. When two-particle states are involved, we take the convention of Jacob and Wick [33] where particle 1 is described by the spinors $u_{\lambda}^{(\rho)}(\mathbf{p})$, while for particle 2 the spinors $u_{-\lambda}^{(\rho)}(-\mathbf{p})$ are used. The two-particle states built from these helicity spinors form a complete basis $\left|\mathbf{p} \lambda_{1} \lambda_{2} \rho\right\rangle$ (with $\left.\rho=\left(\rho_{1}, \rho_{2}\right)\right)$ in Dirac space. In this representation the t-matrix is given by

$$
<\mathbf{p}_{f} \lambda_{1}^{\prime} \lambda_{2}^{\prime} \rho^{\prime}\left|\phi\left(p_{f}, p, P\right)\right| \mathbf{p} \lambda_{1} \lambda_{2} \rho>
$$

Its angular dependence can be exhibited using the total angular momentum states

$$
\left|J M \lambda_{1} \lambda_{2}>=\sqrt{\frac{2 J+1}{2}} \int d \Omega_{p} D_{M \lambda}^{J^{*}}(\theta, \varphi)\right| p, \theta, \varphi, \lambda_{1}, \lambda_{2}>.
$$

In view of rotational invariance of the t-matrix, Eq. (A8) can be written in the form

$$
t \equiv \sum_{n} \phi_{n ; m^{\prime}, m}\left(p_{f}, p\right)=\sum_{J, M} \frac{2 J+1}{2} D_{M \lambda^{\prime}}^{J^{*}}\left(\Omega_{f}\right) \phi_{m^{\prime}, m}^{J}\left(p_{f}, p\right) D_{M \lambda}^{J}(\Omega)
$$

where we have defined $\phi_{m^{\prime}, m}^{J}\left(p_{f}, p\right)=<p_{f} J \lambda_{1}^{\prime} \lambda_{2}^{\prime} \rho^{\prime}\left|\phi\left(p_{f}, p, P\right)\right| p J \lambda_{1} \lambda_{2} \rho>$ with $n=\{J, M\}, m^{\prime}=\left\{\lambda_{1}^{\prime}, \lambda_{2}^{\prime}, \rho^{\prime}\right\}$ and $m=\left\{\lambda_{1}, \lambda_{2}, \rho\right\}$. These angular momentum states can be related to states labeled by the set $\{J M L S\}$. We have

$$
\begin{aligned}
t=\sum_{J, M} & \sum_{L^{\prime}, S^{\prime}, L, S} \sqrt{\frac{2 L^{\prime}+1}{2}} \sqrt{\frac{2 L+1}{2}} \\
& \times D_{M \lambda^{\prime}}^{J^{*}}\left(\Omega_{f}\right) C_{0 \lambda^{\prime} \lambda^{\prime}}^{L^{\prime} S^{\prime} J} D_{M \lambda}^{J^{*}}\left(\Omega_{p}\right) C_{0 \lambda \lambda}^{L S J}<J L^{\prime} S^{\prime}\left|\phi^{J}\left(p_{f}, p, P\right)\right| J L S>
\end{aligned}
$$

A detailed analysis is given in the Refs. [6, 14, 32]. The free np pair can be described by the two-particle states $\left|\mathbf{p} \lambda_{1} \lambda_{2}\right\rangle$ with $\rho=(+,+)$. These helicity states are connected to the total spin $S$ states $\mid \mathbf{p}, S, M_{S}>$ through the relation

$$
\left|\mathbf{p}, S, M_{S}>=\sum_{\lambda_{1} \lambda_{2}} D_{M_{S} \lambda}^{S^{*}}\left(\Omega_{p}\right) C_{\lambda_{1}-\lambda_{2} \lambda}^{\frac{1}{2} \frac{1}{2} S}\right| \mathbf{p} \lambda_{1} \lambda_{2}>
$$




\section{APPENDIX B: THE $K_{0}$ LOOP INTEGRATION IN THE E.M. CURRENT}

In the current operator for the IA in the equal time approximation, initial and final state are assumed to be independent of the relative energy variable. As a result the only dependence on this variable is in the nucleon propagators. Let us first consider the inelastic case. The integral we want to study is of the form

$$
I=\int d k_{0}^{\prime} S_{2}\left(k^{\prime}, P^{\prime}\right) \tilde{\Gamma}_{\mu} S^{(1)}(k, P)
$$

where $\tilde{\Gamma}_{\mu}$ is the e.m. operator including the boost operators. Both sets of variables $\left(k^{\prime}, P^{\prime}\right)$ and $(k, P)$ are in their own c.m. frame, i.e. $P^{\prime}=\left(\mathbf{0}, 2 E^{\prime}\right)$ and $P=(\mathbf{0}, 2 E)$ where $2 E^{\prime}=M_{n p}$ and $2 E=M_{D}$. The Lorentz transformation $\mathcal{L}$ of the c.m. frame to the lab frame is given by Eq. (2.9).

To perform the $k_{0}^{\prime}$ integration we have to analyze the position of the singularities in the propagators. The singularities in the two-particle propagator $S_{2}\left(k, P^{\prime}\right)$ are located at

$$
\begin{aligned}
& (1) k_{0}^{\prime}=E^{\prime}+E_{\mathbf{k}^{\prime}}-i \varepsilon,(2) k_{0}^{\prime}=-E^{\prime}+E_{\mathbf{k}^{\prime}}-i \varepsilon \\
& (3) k_{0}^{\prime}=E^{\prime}-E_{\mathbf{k}^{\prime}}+i \varepsilon,(4) k_{0}^{\prime}=-E^{\prime}-E_{\mathbf{k}^{\prime}}+i \varepsilon
\end{aligned}
$$

while in the initial one-particle propagator $S^{(1)}\left(k, P^{c m}\right)$, expressed in the final state variable $k^{\prime}$, are given by

$$
\begin{aligned}
& (5) k_{0}^{\prime}=\left(\omega^{c m}-E^{\prime}\right)+E_{\mathbf{k}^{\prime}-\mathbf{q}}^{c m}-i \varepsilon, \\
& (6) k_{0}^{\prime}=\left(\omega^{c m}-E^{\prime}\right)-E_{\mathbf{k}^{\prime}-\mathbf{q}}^{c m}+i \varepsilon .
\end{aligned}
$$

In the breakup region $E>M_{N}$ a pinching can occur between the poles $k_{0}^{\prime(2)}$ and $k_{0}^{\prime(3)}$. This generates the elastic cut defined by $E_{\mathbf{k}^{\prime}}=E^{\prime}$. Moreover, the possibility exists that the singularities $k_{0}^{\prime(2)}$ and $k_{0}^{\prime(5)}$ may cross. This occurs when

$$
E_{\mathbf{k}^{\prime}-\mathbf{q}}^{c m}-E_{\mathbf{k}^{\prime}}=-\omega^{c m}
$$

In Fig. 15 is shown the kinematic region of $q$ and $\omega$ where this can happen. The solid line is for small $\omega$ given by $\omega \approx q^{l a b^{2}} / 2 M_{N}$ which corresponds to quasielastic scattering. Note that for real photon absorption $\omega=q$ this double pole doesn't occur. The condition (B4) corresponds to the situation when the hit particle before and after the absorption of the photon is on the mass shell. Since these poles are on the same side of the contour, their crossing doesnot correspond to a pinching singularity.

By closing the contour in for instance the lower half plane the integral over $k_{0}^{\prime}$ can be performed. The integral picks up contributions from the poles at $k_{0}^{\prime(1)}, k_{0}^{\prime(2)}$ and $k_{0}^{\prime(5)}$. The singularity at $k_{0}^{\prime(1)}$ is a negative energy state contribution, the other two are from positive energy states. In so doing we get

$$
\begin{aligned}
I= & 2 \pi i \Lambda_{2}^{-}\left(\mathbf{k}^{\prime}\right) S^{(2)}\left(k^{(1)}, P^{\prime}\right) \tilde{\Gamma}_{\mu} S^{(1)}\left(k^{(1)}, P\right) \\
& -2 \pi i \Lambda_{1}^{+}\left(\mathbf{k}^{\prime}\right) S^{(2)}\left(k^{\prime(2)}, P^{\prime}\right) \tilde{\Gamma}_{\mu} S^{(1)}\left(k^{(2)}, P\right) \\
& -2 \pi i S^{(1)}\left(k^{\prime(5)}, P^{\prime}\right) S^{(2)}\left(k^{(5)}, P^{\prime}\right) \tilde{\Gamma}_{\mu} \Lambda_{1}^{+}(\mathbf{k}) \frac{E_{\mathbf{k}}}{E_{\mathbf{k}^{\prime}-\mathbf{q}}^{c m}}
\end{aligned}
$$

where the four vectors $k^{(i)}$ and $k^{(i)}$ have as fourth component $k_{0}^{(i)}$ and $k_{0}^{\prime(i)}$ respectively. If we consider only positive energy intermediate states Eq. (B1) reduces to

$$
\begin{aligned}
I= & -2 \pi i \Lambda_{1}^{+}\left(\mathbf{k}^{\prime}\right) \Lambda_{2}^{+}\left(\mathbf{k}^{\prime}\right) \tilde{\Gamma}_{\mu} \Lambda_{1}^{+}(\mathbf{k}) \\
& {\left[\frac{1}{E+k_{0}^{(2)}-E_{k}} \frac{1}{2\left(E^{\prime}-E_{\mathbf{k}^{\prime}}\right)}+\frac{1}{E^{\prime}+k_{0}^{\prime(5)}-E_{\mathbf{k}^{\prime}}} \frac{1}{E^{\prime}-k_{0}^{\prime(5)}-E_{\mathbf{k}^{\prime}}} \frac{E_{\mathbf{k}}}{E_{\mathbf{k}^{\prime}-\mathbf{q}}}\right] . }
\end{aligned}
$$

It should be noted, that both contributions develop separately a double pole for $\mathbf{k}$ satisfying Eq. (B4). However, the two terms cancel at this point leading to only a single order pole. Furthermore notice that only the first term has a pole of the form $\left(E^{\prime}-E_{k^{\prime}}\right)^{-1}$ which generates the elastic cut.

In elastic scattering we take as integration variable $k_{0}$ and the the vertex $\tilde{\Gamma}_{\mu}$ is evaluated in the Breit frame. In terms of the variable $k_{0}$ the singularities (B2, B3) are given by 


$$
\begin{aligned}
& (1) k_{0}=E+E_{\mathbf{k}}-i \varepsilon \\
& (2) k_{0}=-\omega-E+E_{\mathbf{k}+\mathbf{q}}-i \varepsilon \\
& (3) k_{0}=E-E_{\mathbf{k}}+i \varepsilon \\
& (4) k_{0}=-\omega-E-E_{\mathbf{k}+\mathbf{q}}+i \varepsilon \\
& (5) k_{0}=-E+E_{\mathbf{k}}-i \varepsilon \\
& (6) k_{0}=-E-E_{\mathbf{k}}+i \varepsilon .
\end{aligned}
$$

It can readily be shown that the kinematics in this case is such that the poles $k_{0}^{(2)}$ and $k_{0}^{(5)}$ cannot cross. Closing the contour in the lower half plane, the integral becomes

$$
\begin{aligned}
I_{\text {elastic }}= & 2 \pi i S^{(1)}\left(k^{\prime(1)}, P^{\prime}\right) \tilde{\Gamma}_{\mu} S^{(1)}\left(k^{(1)}, P\right) \Lambda_{2}^{-}(\mathbf{k}) \\
& -2 \pi i S^{(1)}\left(k^{\prime(5)}, P^{\prime}\right) \tilde{\Gamma}_{\mu} \Lambda_{1}^{+}(\mathbf{k}) S^{(2)}\left(k^{(5)}, P\right) \\
& -2 \pi i \frac{E_{\mathbf{k}^{\prime}}}{E_{\mathbf{k}+\mathbf{q}}} \Lambda_{1}^{+}\left(\mathbf{k}^{\prime}\right) \tilde{\Gamma}_{\mu} S^{(1)}\left(k^{(2)}, P\right) S^{(2)}\left(k^{(2)}, P\right) .
\end{aligned}
$$

* Present address: Philips Research Laboratories, Prof. Holstlaan 4, 5656 AA Eindhoven, The Netherlands.

[1] M. van der Schaar et al., Phys. Rev. Lett. 66, 2855 (1991); ibid. 69, 776 (1992).

[2] M. van der Schaar, Ph.D. thesis, University of Utrecht, 1991 (unpublished).

[3] J-E. Ducret, private communication.

[4] B. Parker et al, Phys. Rev. C34, 2354 (1986).

[5] S. Dytman et al., Phys. Rev. C38, 800 (1988); and private communication.

[6] J. Fleischer and J.A. Tjon, Nucl. Phys. B84,375 (1975); Phys. Rev. D15, 2537 (1977); ibid. D21, 87 (1980).

[7] M.J. Zuilhof and J.A. Tjon, Phys. Rev. C22, 2369 (1980).

[8] E. Hummel and J.A. Tjon, Phys. Rev. C42, 423 (1990).

[9] R. Blankenbecler and R. Sugar, Phys. Rev. 142, 1051 (1966).

[10] A.A. Logunov and A.N. Tavkhelidze, Nouvo Cim.29, 380 (1963).

[11] F. Gross and D.O. Riska, Phys. Rev. C36, 1928 (1987).

[12] J.D. Björken and S.D. Drell, Relativistic Quantum Fields, McGraw-Hill (1965).

[13] T.W. Donnelly and A.S. Raskin, Ann. Phys. 169, 247 (1986).

[14] J.J. Kubis, Phys. Rev. D6, 547 (1972).

[15] J.A. Tjon, Relevance of Relativity in Nuclei, in Hadronic Physics with multi-GeV Electrons, Les Houches Series, Nova Science Publ (New York), page 89.

[16] R.A. Arndt, Phys. Rev. D35, 128 (1987).

[17] M.J. Zuilhof, Ph.D. thesis, University of Utrecht, 1981 (unpublished).

[18] M.J. Zuilhof and J.A. Tjon, Phys. Rev. C24, 736 (1981).

[19] G. Höhler, E. Pietarinen, I. Sabba-Stefanescu, F. Barkowski, G.G. Simon, V.H. Walther and R.D. Wendling, Nucl. Phys. B114, 505 (1976).

[20] I. The et al, Phys. Rev. Lett. 67, 173 (1991).

[21] R.G. Arnold, B.T. Chertok, E.B. Dally, A. Grigorian, C.L. Jordan, W.P. Schütz, R. Zdarko, F. Martin and B.A. Mecking, Phys. Rev. Lett. 35, 776 (1975).

[22] S. Auffret et al, Phys. Rev. Lett. 54, 649 (1985).

[23] M.E. Schulze et al, Phys. Rev. Lett. 52, 597 (1984).

[24] R. Gilman et al, Phys. Rev. Lett. 65, 1733 (1990).

[25] J.F. Mathiot, Nucl. Phys. A412, 201 (1984); Phys. Rep. 173, 63 (1989).

[26] B. Sommer, Nucl. Phys. A308, 263 (1978).

[27] W. Leidemann and H. Arenhövel, Nucl. Phys. A393, 385 (1983).

[28] B. Mosconi and P. Ricci, Nucl. Phys. A517, 483 (1990); B. Mosconi et al, Few Body Systems, Suppl. 5, 223 (1992).

[29] G. Beck and H. Arenhövel, Few Body Systems 13, 165 (1992).

[30] E. van Meijgaard and J.A. Tjon Phys. Rev. C45, 1463 (1992).

[31] F. Iachello, A.D. Jackson and A. Landé, Phys. Lett. 43B, 191 (1973).

[32] J.A. Tjon and S.J. Wallace, Phys. Rev. C32, 1667 (1985).

[33] M. Jacob and G.C. Wick, Ann. Phys. 7, 404 (1959). 
FIG. 1. The phase shifts for the BSLT equation as a function of the lab energy for the fits given in Table I. The solid lines are the calculated phase shifts for fit B with the negative energy intermediate states included. The dashed and dotted-dashed lines correspond to fits A from Ref. [6] and B respectively where only positive energy intermediate states have been kept. The data are from Ref. 16 .

FIG. 2. The PWIA ( $\mathrm{a}$ and $\mathrm{b}$ ) and the Born (c and d) contributions to the deuteron current.

FIG. 3. Feynman diagrams corresponding to the IA contribution to the deuteron current with FSI included.

FIG. 4. The charge form factor $F_{C}$ and the quadrupole form factor $F_{Q}$ in the relativistic impulse approximation using the ET and the BSLT prescription for the deuteron current. The data are from Ref. 20].

FIG. 5. The same as Fig. 4, but for the magnetic form factor $F_{M}$.

FIG. 6. The full predictions using the ET and the BSLT prescriptions for the deuteron current with and without MEC contributions, but for the electric and magnetic form factors A and B. The data are from Refs. 21. and 22.

FIG. 7. The same as Fig. 6, but for the tensor polarization $t_{20}$. The data are from Refs. 20,23, 24].

FIG. 8. The relative difference $\frac{R_{\alpha}^{a}\left(\Gamma_{e m}\right)-R_{\alpha}\left(\Gamma_{e m}\right)}{0.01 \times R_{\alpha}\left(\Gamma_{e m}\right)}$ of the response functions $R_{\alpha}$ without negative energy state contributions and $R_{\alpha}^{a}$ calculated in various approximations. When $R^{a}$ is the full result we find the solid curve. Evaluating $R^{a}$ by neglecting both the negative energy states and the boost transformations yields the double dot-dash line. Dropping furthermore also the FSI contributions in $R^{a}$ yields the long dashed curve. The effect of the BSLT propagator choice is given by the dashed curve. The dotted and dot-dashed curves correspond to the results of the relative change for the various NR approximations 7.4 and 7.6 to the current.

FIG. 9. The relative contribution of FSI to the four response functions as function of $q$ and $\cos (\theta)$.

FIG. 10. The relative contribution of negative energy states to the four response functions as function of $q$ and $\cos (\theta)$.

FIG. 11. The response functions for the exclusive Nikhef kinematics. The data are from Ref. [四].

FIG. 12. Longitudinal and transverse response functions for $\mathrm{q}=300 \mathrm{MeV} / \mathrm{c}$. The data are from Ref. [5].

FIG. 13. Longitudinal and transverse response functions for $\mathrm{q}=400 \mathrm{MeV} / \mathrm{c}$. The data are from Ref. [5].

FIG. 14. Longitudinal and transverse response functions for $\mathrm{q}=500 \mathrm{MeV} / \mathrm{c}$. The data are from Ref. [5].

FIG. 15. In the region I there is a crossing between two positive energy poles of the one-particle propagator were before and after absorption of the photon the particle is on mass shell. In region II this crossing doesn't occur. 